RESEARCH ARTICLE

\title{
Result Analysis of BRAF V600E Gene Mutation Using Molecular and Immunohistochemistry Detection in Acral Malignant Melanoma
}

\section{Hermin Aminah Usman ${ }^{1}$, Bethy $S$ Hernowo ${ }^{2}$, Maringan Diapari Lumban Tobing ${ }^{2}$, Reti Hindritiani ${ }^{2}$}

${ }^{1}$ Anatomical Pathology Department, Faculty of Medicine, Universitas Padjadjaran/ Hasan Sadikin General Hospital, Bandung, Indonesia. ${ }^{2}$ Padjadjaran University Bandung.

\begin{abstract}
Background and Objective: Detection of BRAF $\mathrm{V} \neg 600 \mathrm{E}$ gene mutation in Malignant Melanoma (MM) is essential in offering anti BRAF targeted therapy for MM patients. Expression of BRAF V600E mutant protein detected using immunohistochemistry (IHC) is affordable, rapid, and reliable. This study aimed to measure the sensitivity and specificity of IHC compared to BRAFV 600E immunoexpression by molecular detection. Method: This study used a paraffin block of acral MM patients to analyze BRAF V600E mutation status using anti-BRAF V600E IHC compared to Real-Time Polymerase Chain Reaction (RT PCR) which is a gold standard. Result: In total, ninety-six samples were tested using IHC and RT-PCR. RT-PCR showed that $15(15.6 \%)$ samples were positive for BRAF V600E mutation. The sensitivity and specificity for IHC were $73.3 \%$ and $87.6 \%$ respectively. Concordance value of IHC was $85.4 \%$. There were 10 false positives and 4 false negatives. IHC staining may be applied as early detection of BRAFV600E mutation in MM patients that are going to receive anti BRAF targeted therapy.
\end{abstract}

Keywords: BRAF V600E- immunohistochemistry- melanoma- RT-PCR

Asian Pac J Cancer Care, 3 (3), 43-45

\section{Introduction}

Malignant Melanoma (MM) is a malignancy with a high mortality rate due to high metastasis ability and resistance towards chemotherapy and radiotherapy treatment [1-2]. Five years survival rate of metastasis MM was around 20\%. The incidence of MM in the Asian population is lower compared to the Caucasian population. [1-3-4]. The most common histopathology identified in Asian population is Acral Lentigenous Melanoma (ALM) [1]. ALM is the aggressive type of MM with poor prognosis compared to other types of MM [1].

BRAF V600E mutation can cause a deep invasion of malignant melanoma and lead to metastasize. Currently, there are two targeted therapies that could inhibit the effect of BRAF V600E mutation, including vemurafenib and dabrafenib [5]. Before initiating the targeted therapy, at first BRAF gene mutation should be detected in a rapid
Submission Date: 06/09/2018 Acceptance Date: 08/13/2018

and accurate manner so that the targeted therapy could give an optimum result [5].

There are several molecular methods that could be used routinely in detecting BRAF gene mutations, such as Sanger sequencing, pyrosequencing, real-time-polymerase chain reaction (RT-PCR), and high-resolution melting-PCR technique [6]. RT-PCR is the gold standard method for detecting BRAF gene mutation, however, it highly depends on the adequate volume of the tumor mass [6]. Immunohistochemistry has more advantages in detecting BRAF gene mutation since the test only need the minimum volume of the tumor mass. Other than that, the antibody used to detect BRAF V600E protein has been well developed and already sold commercially[6].

This study aims to measure the effectiveness of the immunohistochemistry test for detecting BRAF V600E in malignant melanoma patients which is not already available in Indonesia. 


\section{Materials and Methods}

\section{Objects and Method}

This study used preserved paraffin block of patients diagnosed with acral MM at the Department of Anatomy Pathological in Faculty of Medicine Universitas Padjadjaran/Hasan Sadikin General Hospital from 2011-2016. There were 96 paraffin block samples qualified based on the inclusion criteria. The inclusion criteria of this study are all acral malignant melanoma cases taken from biopsy or operation samples that have been diagnosed histopathologically with a good condition of paraffin block samples and contain enough tumor mass. The steps of the technique used in this study were the detection of BRAF mutation with RT-PCR as molecular technique and detect mutant BRAF V600E protein using immunohistochemistry technique. Ethical clearance was approved by the Health Research Ethics Committee, number 1155/UN6.C1.3.2/KEPK/PN/2016.

\section{BRAF V600E Mutation Detection}

DNA samples were obtained from preserved paraffin block were excised serially into $6 \mu \mathrm{m}$ width in 2 slices. DNA was extracted using AmoyDx1 FFPE DNA Kit (Amoy Diagnostics, China), followed the instruction as described by the manufacturer. DNA refraction on samples was measured using spectrometer A260/A280 with a value between 1.8-2.0. DNA samples were stored at -200C and analyzed by RT-PCR. BRAF V600E mutation status of MM was determined by AmoyDx1 BRAF V600E Mutation Detection Kit (Amoy Diagnostics, China). The amplification of the BRAFV600E gene was using a specific primary antibody and is detected by probes instrument of Bio-Rad CFX96 (Bio-Rad Laboratories, USA). Mutation status was evaluated using fluorescence signal from Carboxyfluorescein (FAM) if the $\mathrm{Ct}$ value $>28$ the result is considered negative whereas FAM Ct value $>28$ is considered positive.

\section{Immunohistochemistry Staining}

Immunohistochemistry staining on the samples was manually performed by labeled streptavidin-biotin immunoperoxidase complex method using Starr Trek Universal HRP Detection system (Biocare Medical,). Samples were excised serially at $4 \mu \mathrm{m}$ width. Antigen retrieval used the decloaking instrument for 45-60 minutes in $980 \mathrm{C}$. Primary antibody used was anti-
BRAF V600E (Revmab, 1:500 Bioscience USA). Immunoexpression of BRAFV600E in the nuclear was assessed on semi-quantitative scores based on the intensity and distribution of positive cells. Intensity scores were negative (0), weakly positive (1), moderate positive (2), and strongly positive (3). The percentage of positive cells: $0=0 \%, 1=<25 \%, 2=26-50 \%, 3=51-75 \%$, $4=76 \%-100 \%$. The final score was calculated by histoscore, namely intensity $\mathrm{X}$ distribution with scores 0-6 regarded as negative, 8-12 were stated as positive.

\section{Results}

About 15 samples (15.6\%) were positive for BRAF V600E gene mutation (Graph 3.1). Negative BRAF V600E mutation was found in 81 samples $(84.4 \%)$. There were 10 samples with false positives result (positive in IHC, negative in RT-PCR) and 4 samples with the false negative result (negative in IHC, positive in RT-PCR). The sensitivity was $73,3 \%$, specificity was $87,6 \%$, and concordance value was $85,4 \%$.

\section{Discussion}

The purpose of this study was to measure the sensitivity and specificity of IHC compared with molecular technique to detect BRAF V600E gene mutation. this study showed that the sensitivity and specificity of IHC were $73,3 \%$ and $87,6 \%$ respectively. These results were lower than the previous studies. In that previous studies, the antibody used was clone V1 which is commercially available. However, the antibody used in this study was Revmab clone [5-7-10]. Different antibody clone may result in different expression. It also occurred in the study by Liu et al that reports a sensitivity of $72.2 \%$ [11].

This study showed 10 false positives in tumor sample that contains more than $25 \%$ of melanin pigment. This pigment could disguise protein expression in tumor cells result in the protein expression seem stronger (immunopositive) or ambiguous. Kevin et al studied that tumor mass with more than $10 \%$ melanin pigment has caused different measurement among pathologist. [12]. This study utilized 3,3'-diaminobenzidine (DAB) as the chromogen to determine protein expression. DAB chromogen stained brown similar to melanin pigment. To avoid ambiguity,3-amino-9-ethylcarbazole (AEC) chromogen should be used to stain red in expressing

Table 3.1. Comparison of BRAF V600E Gene Mutation

\begin{tabular}{lccc}
\hline & & BRAF V600E mutation (RT-PCR) & Total \\
\hline IHC of BRAFV600E & Positive & Negative & 21 \\
Positive & 11 & 10 & 75 \\
Negative & 4 & 71 & 96 \\
Total & 15 & 81 & Concordance* \\
& Sensitivity & Specificity & $85.4 \%$ \\
\hline
\end{tabular}

\footnotetext{
*Concordance, (true positive+true negative)/whole sample number.
} 
the protein as seen in Thiel et al study [6]. This was the limitation of this study due to the availability of DAB chromogen only. Other than melanin pigment, factors that could disguise protein expression in malignant melanoma are the macrophage, wide necrosis, and tumor mass in traumatic samples [5-6].

The most common BRAF gene mutation found in MM was V600E gene mutation (73-74\%), followed by V600K mutation (19-20\%) [5]. IHC technique to detect BRAF V600E mutation may exhibit cross-reactivity with BRAF V600K mutation. This cross-reactivity is due to the epitope similarity of BRAF V600E and BRAF V600K or both the BRAF mutation present in the tumor mass. To overcome these problems, we still need to use RT-PCR test to confirm which mutation present in the tumor mass $[5,13]$.

Several studies found heterogeneity in tumor mass may exhibit false expression, whether positive or negative. A false expression can be eluded if the mutation was detected through molecular examination such as pyrosequencing, Sanger sequencing, or high-resolution melting analysis[6-8-14-15].

Four false negatives in this study were caused by inadequate intervention during the pre-analytic phase [13]. The optimal tissue fixation is using neutral $10 \%$ formalin buffer for 12-24 hours. Fixation with this solution for less than 12 hours or using other solutions may result in weaker expression in IHC staining [13]. The laboratory used in this study was performed using standardized fixation solution, but suboptimal fixation cannot be excluded that resulted in false negative results.

Prior to the administration of targeted therapy in malignant melanoma patients, determination of BRAFV600E is essential. The gold standard to detect the mutation is a molecular technique. However, this molecular detection has several disadvantages such as takes a longer test period, more expensive and require adequate tumor mass to obtain an accurate result. On the other hand, IHC is more reliable, cheaper, and gives good result even with minimum tumor mass. This study showed that the IHC test of BRAFV600E has specificity more than $85 \%$ with NPV around $97,2 \%$. Thus, several things should be addressed: specific caution should be performed on tumor mass with abundant melanin pigments since they could alter the expression measurement, and tissue preparation should also follow the standard procedure to get optimum sample fixation.

The result of this study is that IHC can be used to detect BRAFV600E mutation as the early screening test before detected by the molecular technique is performed in malignant melanoma patients that are going to receive anti BRAF targeted therapy. Further study with larger MM samples by cohort design may give higher specificity and sensitivity value.

\section{References}

1. Hong JW, Lee S, Kim DC, Kim KH, Song KH. Prognostic and Clinicopathologic Associations of BRAF Mutation in Primary Acral Lentiginous Melanoma in Korean Patients:
A Preliminary Study. Annals dermatol. 2014;26:195-202.

2. Qiu T, Lu H, Guo L, Huang W, Ling Y, Shan L. Detection of BRAF mutation in Chinese tumor patients using a highly sensitive antibody immunohistochemistry assay. Scientific Reports. 2015;5:9211.

3. Kim HK, Lee S, Kim K, Heo MH, Lee H, Cho J. Efficacy of BRAF Inhibitors in Asian Metastatic Melanoma Patients: Potential Implications of Genomic Sequencing in BRAFMutated Melanoma. Translational Oncol.2016;9 :557-64.

4. Asgari MM, Shen L, Sokil MM, Yeh I, Jorgenson E. Prognostics factors and survival in acral lentiginous melanoma. British J Dermatol. 2017 Aug;177:428-435.

5.Huang WK, Kuo TT, Wu CE, Cheng HY, Hsieh CH, Hsieh JJ. A comparison of immunohistochemical and molecular methods used for analyzing the BRAF V600E gene mutation in malignant melanoma in Taiwan. Asia-Pacific J Clin Oncol. 2016;12:403-8.

6.Thiel A, Moza M, Kytola S, Orpana A, Jahkola T, Hernberg $M$. Prospective immunohistochemical analysis of BRAF V600E mutation in melanoma. Hum Pathol. 2015;46:169-75.

7.Marin C, Beauchet A, Capper D, Zimmermann U, Julié C, Ilie M. Detection of BRAF p.V600E Mutations in Melanoma by Immunohistochemistry Has a Good Interobserver Reproducibility. Archives Pathol Lab Med. 2013;138:71-5.

8.Colomba E, Helias-Rodzewicz Z, Von Deimling A, Marin C, Terrones N, Pechaud D. Detection of BRAF p.V600E mutations in melanomas: comparison of four methods argues for sequential use of immunohistochemistry and pyrosequencing. J Mol diag : JMD. 2013;15:94-100.

9.Sun J, Zhang J, Lu J, Gao J, Lu T, Ren X. Immunohistochemistry is highly sensitive and specific for detecting the BRAF V600E mutation in papillary thyroid carcinoma. Int J Clin Exp Pathol.2015;8:15072-8.

10.Feller JK, Yang S, Mahalingam M. Immunohistochemistry with a mutation-specific monoclonal antibody as a screening tool for the BRAFV600E mutational status in primary cutaneous malignant melanoma. Mod Pathol.2013;26:414-20.

11.Liu H, Li Z, Wang Y, Feng Q, Si L, Cui C. Immunohistochemical detection of the BRAF V600E mutation in melanoma patients with monoclonal antibody VE1. PatholInt. 2014;64:601-6.

12.Fisher KE, Cohen C, Siddiqui MT, Palma JF, Lipford EH, 3rd, Longshore JW. Accurate detection of BRAF p.V600E mutations in challenging melanoma specimens requires stringent immunohistochemistry scoring criteria or sensitive molecular assays. Hum Pathol. (2014) Nov;45(11):2281-93.

13.Tetzlaff MT, Pattanaprichakul P, Wargo J, Fox PP, Patel KP, Estrella JS. Utility of BRAF V600E immunohistochemistry expression pattern as a surrogate of BRAF mutation status in 154 patients with advanced melanoma. HumPathol. (2015) ;46(8):1101-10.

14.Yaman B, Kandiloglu G, Akalin T. BRAF-V600 Mutation Heterogeneity in Primary and Metastatic Melanoma: A Study With Pyrosequencing and Immunohistochemistry. Am J Dermatopathol. (2016) Feb;38(2):113-20.

15.Bruno W, Martinuzzi C, Andreotti V, Pastorino L, Spagnolo F, Dalmasso B. Heterogeneity and frequency of BRAF mutations in primary melanoma: Comparison between molecular methods and immunohistochemistry. Oncotarget. (2017) Jan 31;8(5):8069-82.

This work is licensed under a Creative Commons AttributionNon Commercial 4.0 International License. 